\title{
A broad expression profile of the GMR-GAL4 driver in Drosophila melanogaster
}

\author{
W.-Z. Li, S.-L. Li, H.Y. Zheng, S.-P. Zhang and L. Xue \\ Shanghai Key Laboratory for Signaling and Diseases, \\ School of Life Science and Technology, Tongji University, Shanghai, China \\ Corresponding author: L. Xue \\ E-mail: 1ei.xue@tongji.edu.cn
}

Genet. Mol. Res. 11 (3): 1997-2002 (2012)

Received July 20, 2011

Accepted January 6, 2012

Published August 6, 2012

DOI http://dx.doi.org/10.4238/2012.August.6.4

\begin{abstract}
The GAL4/UAS binary system has been widely used in Drosophila melanogaster for ectopic expression of transgenes in a tissue-specific manner. The GMR-GAL4 driver, which expresses the yeast transcription factor GAL4 under the control of glass multiple reporter $(G M R)$ promoter elements, has been commonly utilized to express target transgenes, specifically in the developing eye. However, we have observed abnormal wing phenotypes; this is a result of the activity of critical wing developing genes, e.g., components of the Notch or Wg pathway, that are up- or down-regulated under the control of the GMR-GAL4 driver. X-gal staining confirmed that $U A S$-LacZ is expressed in third-instar larva wing imaginal discs, as well as in eye discs, when driven by the GMR-GAL4 driver. Furthermore, we found that $G M R$-GAL4 also drives $U A S$-LacZ expression in other tissues, such as brain, trachea, and leg discs. These results indicate that GMRGAL4 has a broad expression profile, rather than the eye-specific pattern described previously, and that one should be careful when using it as a tool for targeted gene expression.
\end{abstract}

Key words: GMR-GAL4; Drosophila; Wing; Expression 


\section{INTRODUCTION}

The GAL4/UAS bipartite expression system has been utilized as an extremely powerful tool to ectopically express transgenes in specific patterns in Drosophila melanogaster (Duffy, 2002; Traven et al., 2006). In this system, the yeast transcription factor GAL4 is driven by various promoter sequences, and thus, exhibits many different temporal and spatial expression profiles (Laughon et al., 1984; Brand and Perrimon, 1993). On the other hand, the transgenes are placed under the control of the GAL4 target sequence, upstream activation sequence $(U A S)$, and could be transcriptionally activated by GAL4 (Brand and Perrimon, 1993). GMR-GAL4 was constructed by Freeman in 1996 and was reported to drive the expression of target genes in all cells posterior to the morphogenetic furrow in the developing eye specifically (Song et al., 2000). Since then, many overexpression studies have been carried out in Drosophila, using the GMR-GAL4 driver line with attention being focused on the developing eyes.

The Notch signaling pathway, which has been highly conserved in most multi-cellular organisms, is important for cell-cell communication (Major and Irvine, 2005). Notch was first identified in Drosophila as an important gene for wing development (de Celis and GarciaBellido, 1994). It is required for the establishment of the dorsal-ventral compartment border, and the proper development of wing margin, veins, and sensory organs (Casso et al., 2011). The Notch receptor is activated by the Delta and Jagged/Serrate families of membrane-bound ligands (Weinmaster, 1997).

The Wnt signaling pathway is another evolutionary conserved pathway that plays pivotal roles in embryogenesis and other physiological processes. Dysfunction of the Wnt pathway leads to many diseases including cancer (Lie et al., 2005). The Drosophila wingless gene $(w g)$ is the founding member of the Wnt family, which was originally identified as a recessive mutation affecting wing and haltere development (Sharma and Chopra, 1976). The Wg protein can bind to cell-surface receptors of the Frizzled family, which activates Dishevelled (Dsh), ultimately resulting in the accumulation of Armadillo (Arm), which reaches the nucleus and subsequently promotes specific gene expression (Benitez et al., 2009).

In this report, we showed that expression of wild-type, dominant negative or RNAi of the critical wing developing genes, e.g., Notch and Wg pathway components, under the control of three independent GMR-GAL4 drivers, results in various abnormal wing phenotypes, suggesting that GMR-GAL4 is expressed in the developing wing. We performed X-Gal staining to examine $U A S$-LacZ expression driven by GMR-GAL4, and confirmed that GMR-GAL4 is expressed in third-instar larval wing discs, as well as in other tissues including brain, trachea and leg discs. These data demonstrate that GMR-GAL4 has a much broader expression pattern than previously described, which should be borne in mind when over-expression analysis is performed using GMR-GAL4 as the driver.

\section{MATERIAL AND METHODS}

\section{Drosophila strains}

GMR-GAL4, UAS-LacZ, UAS-Dl, UAS-N.RNAi, UAS-D1 ${ }^{\mathrm{DN}}, U A S$-Wg, $U A S$-Dsh were obtained from the Bloomington Drosophila Stock Center and $U A S$-wg.RNAi from the VDRC Center. 


\section{X-Gal staining}

Eye and wing discs were dissected from 3rd-instar larvae in PBST and stained for $\beta$-galactosidase activity as previously described (Xue et al., 2007).

\section{RESULTS AND DISCUSSION}

The GMR-GAL4 driver has been commonly used to induce ectopic expression of target genes in the developing eyes, more specifically, in the cells posterior to the morphogenetic furrow in the eye discs (Freeman, 1996). The expression profile of GMR-Gal4 in other tissues, however, has not been well characterized. To address this issue, we expressed Delta (Dl), a ligand of the Notch signaling that plays important roles in the development of most tissues, under the control of GMR-GAL4 (GMR $>\mathrm{Dl})$. Three independent $G M R$-Gal4 lines were tested in this and all subsequent experiments and similar results were obtained.

We found the $G M R>\mathrm{Dl}$ flies not only show the eye phenotype as previously reported (Shalaby et al., 2009; data not shown), but also display a wing phenotype. In most cases, the distal part of vein L5 was lost in the GMR $>\mathrm{Dl}$ wings (Figure 1C and D), as compared to the wild-type control (Figure 1A and B). Since Notch signaling is known to inhibit vein formation and regulate dorsal-ventral patterning in wing development, our finding suggests that $G M R$-Gal4 might be expressed in the developing wings. Consistent with this explanation, downregulation of the Notch pathway by the expression of a Notch RNAi under the control of GMR-Gal4 resulted in extra veins (Figure 1E and F), and expression of a dominant negative form of Notch generated thicker veins (Figure $1 \mathrm{G}$ and $\mathrm{H}$ ), and sometimes water bubbles between dorsal/ventral layers in the wing (Figure 1J, compared to wild type in 1I).

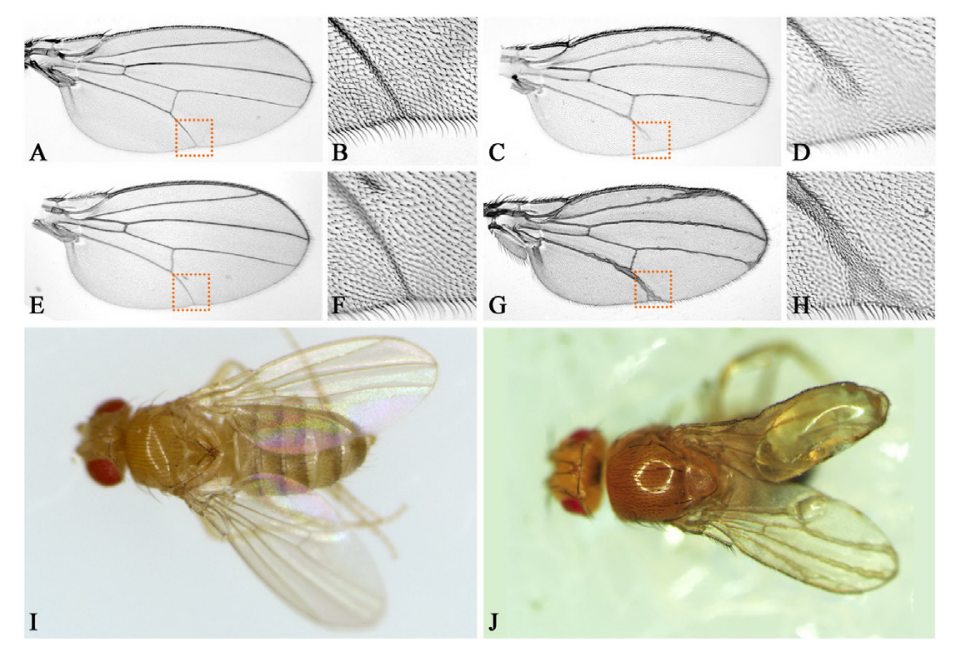

Figure 1. Various wing phenotypes resulting from expression of Notch pathway components driven by GMRGAL4. Compared to a wild-type wing (A, B and I), activation of Notch signaling by GMR-Gal4 inhibits vein L5 formation in the distal area (C and $\mathbf{D}, G M R-G a l 4 / U A S-\mathrm{Dl})$, while downregulation of Notch signaling by GMRGal4 results in extra veins ( $\mathbf{E}$ and $\mathbf{F}, G M R$-Gal4/UAS-N.RNAi), thicker veins ( $\mathbf{G}$ and $\mathbf{H}, G M R$-Gal4/UAS-Dl ${ }^{\mathrm{DN}}$ ), and water bubbles between dorsal/ventral layers $\left(\mathbf{J}, G M R-G a 14 / U A S-\mathrm{N}^{\mathrm{DN}}\right)$. B, D, F, and $\mathrm{H}$ are magnifications of the boxed areas in A, C, E, and G, respectively. 
The Wingless (Wg) signaling pathway is another crucial regulator of Drosophila wing development. While ectopic Wg signaling results in extra vein and bristle formation, insufficient Wg signaling leads to loss of wing tissue (Williams et al., 1993). We found that downregulation of Wg signaling by the expression of a wg.RNAi under the control of GMR-Gal4 results in loss of wing margin tissues (Figure 2C and D), and expression of its downstream component Arm RNAi leads to shrunken wings (Figure $2 \mathrm{~B}$, compare to wild type in $2 \mathrm{~A}$ ). In addition, ectopic expression of $\mathrm{Wg}$ or its downstream component Dsh driven by GMR-Gal4 produced extra veins (Figure 2E and F) and bristles (Figure $2 \mathrm{G}$ and $\mathrm{H}$ ).
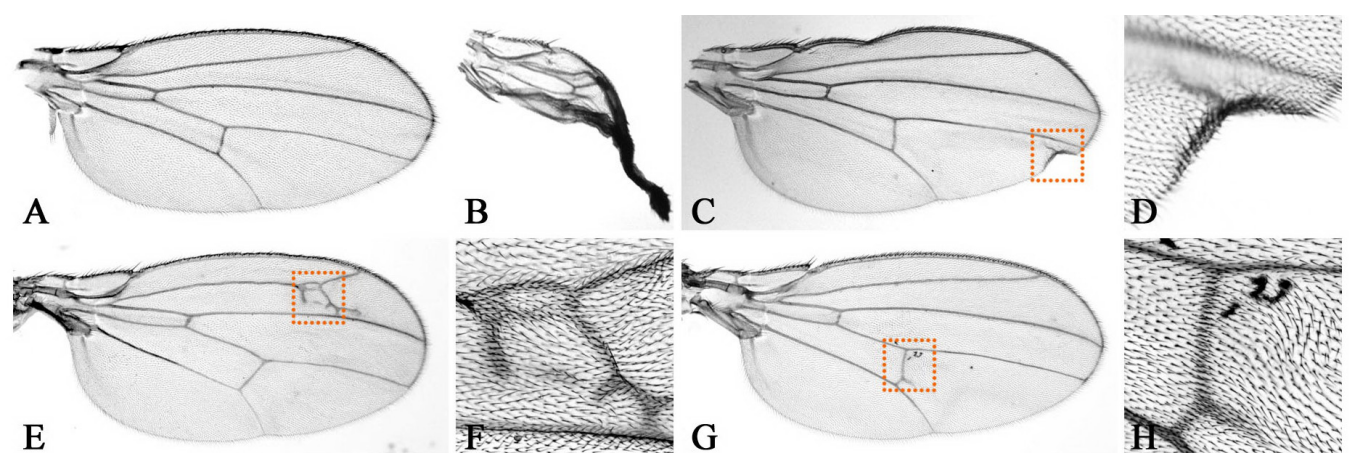

Figure 2. Various wing phenotypes resulting from expression of Wg pathway components driven by GMR-GAL4. Compared to a wild-type wing (A), downregulation of $\mathrm{Wg}$ signaling by $G M R$-Gal4 results in shrunken wing (B, $G M R$-Gal4/UAS-Arm.RNAi), or notch of wing margin (C and D, GMR-Gal4/UAS-Wg.RNAi), while activation of Wg signaling by GMR-Gal4 induces the formation of extra veins ( $\mathbf{E}$ and $\mathbf{F}, G M R$-Gal4/UAS-Wg) or bristles ( $\mathbf{G}$ and H, GMR-Gal4/UAS-Dsh). D, F and $\mathrm{H}$ are magnifications of the boxed areas in $\mathrm{C}, \mathrm{E}$ and $\mathrm{G}$, respectively.

The adult wing phenotypes described above suggest that GMR-Gal4 is most likely also expressed in the developing wing. To confirm this notion, we crossed three independent $G M R$-Gal4 lines to $U A S$-LacZ and examined the expression pattern of LacZ in 3rd-instar larval wing discs. Compared to the wild-type control (Figure 3A), all three GMR-Gal4 lines were able to drive LacZ expression in the wing discs, albeit at different strength (Figure 3B-D). The expression patterns of three GMR-Gal4 lines were partially, but not completely overlapping (Figure 3B-D). As a positive control, LacZ was induced in the eye imaginal discs, most prominently, posterior to the morphogenetic furrow, by all three GMR-Gal4 drivers (Figure 3F-H).

We further investigated the expression pattern of GMR-Gal4 in other tissues, and found LacZ expression in the larval brain (Figure 4D), leg disc (Figure 4E) and trachea (Figure $4 \mathrm{~F}$ ), as compared to the control (Figure 4A-C). Taken together, our results indicate that GMRGal4 has a much broader expression profile, rather than the eye-specific pattern, as commonly regarded. Researchers should be cautious in interpreting certain phenotypes, such as lethality and behavioral defects, resulting from transgene over-expression driven by the GMR-Gal4 driver. 


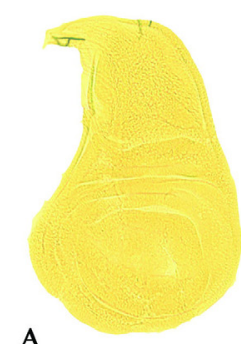

A

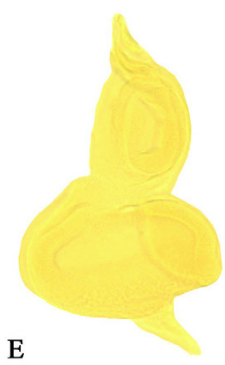

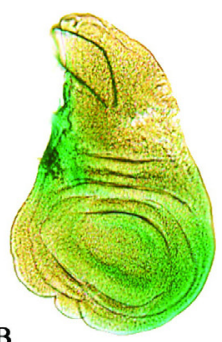

B

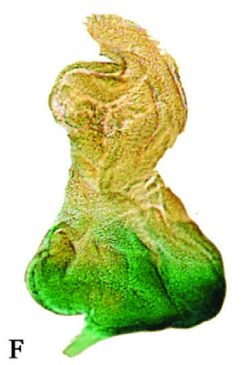

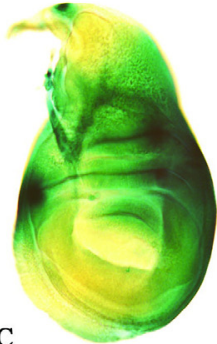

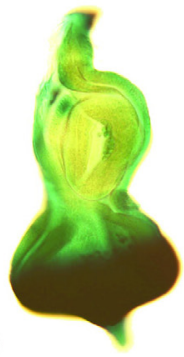

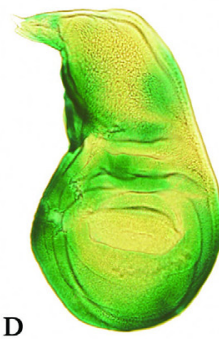

$\mathrm{H}$

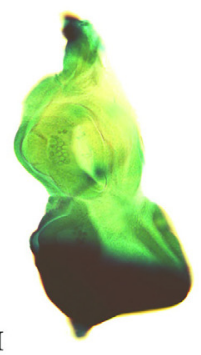

Figure 3. GMR-GAL4 is expressed in wing and eye discs. X-Gal staining of a $U A S$-LacZ reporter gene driven by no (A and $\mathbf{E}$ ) or three independent GMR-GAL4 drivers (B-D, F-H) in 3rd-instar larval wing (A-D) or eye (E-H) discs.

A

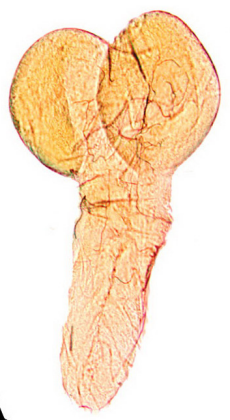

D

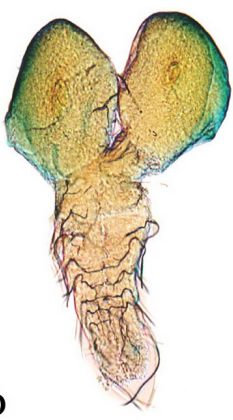

B
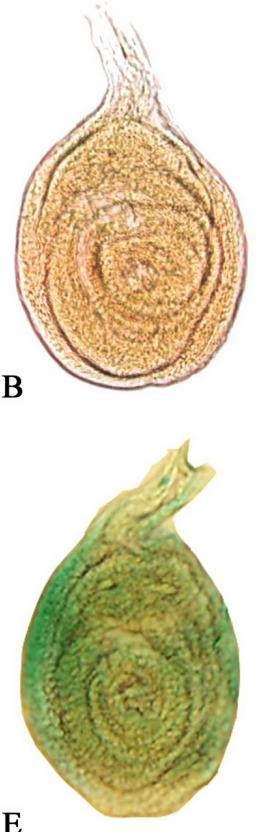

C

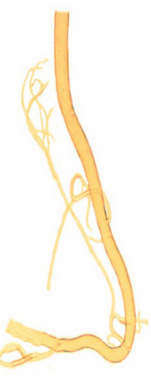

F

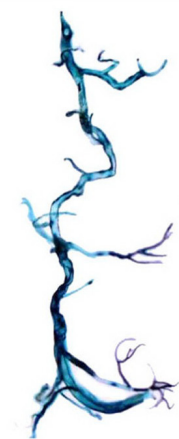

Figure 4. GMR-Gal4 is expressed in other developing tissues. X-Gal staining of a $U A S$-LacZ reporter gene driven by no (A-C) or a GMR-GAL4 driver (D and $\mathbf{E}$ ) in 3rd-instar larval brain (A and D), leg disc (B and $\mathbf{E})$ or trachea $(\mathbf{C}$ and $\mathbf{F})$. 


\section{ACKNOWLEDGMENTS}

Research supported by the National Natural Science Foundation of China (Grant \#30971681, \#31071294), the Innovation Program of Shanghai Municipal Education Commission (Grant \#10ZZ27), the Program for New Century Excellent Talents in University (Grant \#NCET-10-0608) and the Shanghai Committee of Science and Technology (Grant \#09DZ2260100). We thank the Bloomington and VDRC Stock Centers for fly stocks.

\section{REFERENCES}

Benitez E, Bray SJ, Rodriguez I and Guerrero I (2009). Lines is required for normal operation of Wingless, Hedgehog and Notch pathways during wing development. Development 136: 1211-1221.

Brand AH and Perrimon N (1993). Targeted gene expression as a means of altering cell fates and generating dominant phenotypes. Development 118: 401-415.

Casso DJ, Biehs B and Kornberg TB (2011). A novel interaction between hedgehog and Notch promotes proliferation at the anterior-posterior organizer of the Drosophila wing. Genetics 187: 485-499.

de Celis JF and Garcia-Bellido A (1994). Roles of the Notch gene in Drosophila wing morphogenesis. Mech. Dev. 46: 109-122. Duffy JB (2002). GAL4 system in Drosophila: a fly geneticist's Swiss army knife. Genesis 34: 1-15.

Freeman M (1996). Reiterative use of the EGF receptor triggers differentiation of all cell types in the Drosophila eye. Cell 87: 651-660.

Laughon A, Driscoll R, Wills N and Gesteland RF (1984). Identification of two proteins encoded by the Saccharomyces cerevisiae GAL4 gene. Mol. Cell Biol. 4: 268-275.

Lie DC, Colamarino SA, Song HJ, Desire L, et al. (2005). Wnt signalling regulates adult hippocampal neurogenesis. Nature 437: 1370-1375.

Major RJ and Irvine KD (2005). Influence of Notch on dorsoventral compartmentalization and actin organization in the Drosophila wing. Development 132: 3823-3833.

Shalaby NA, Parks AL, Morreale EJ, Osswalt MC, et al. (2009). A screen for modifiers of notch signaling uncovers Amun, a protein with a critical role in sensory organ development. Genetics 182: 1061-1076.

Sharma RP and Chopra VL (1976). Effect of the Wingless (wg1) mutation on wing and haltere development in Drosophila melanogaster. Dev. Biol. 48: 461-465.

Song Z, Guan B, Bergman A, Nicholson DW, et al. (2000). Biochemical and genetic interactions between Drosophila caspases and the proapoptotic genes rpr, hid, and grim. Mol. Cell Biol. 20: 2907-2914.

Traven A, Jelicic B and Sopta M (2006). Yeast Ga14: a transcriptional paradigm revisited. EMBO Rep. 7: 496-499.

Weinmaster G (1997). The ins and outs of notch signaling. Mol. Cell Neurosci 9: 91-102.

Williams JA, Paddock SW and Carroll SB (1993). Pattern formation in a secondary field: a hierarchy of regulatory genes subdivides the developing Drosophila wing disc into discrete subregions. Development 117: 571-584.

Xue L, Igaki T, Kuranaga E, Kanda H, et al. (2007). Tumor suppressor CYLD regulates JNK-induced cell death in Drosophila. Dev. Cell 13: 446-454. 